\title{
Surface wettability enhancement on oxide film coated-steels due to gamma-ray irradiation
}

\author{
Wilson SUSANTO*,**, Tomonori IHARA*, Tatsuya HAZUKU*, \\ Shinichi MOROOKA*** and Sho KANO**** \\ *Tokyo University of Marine Science and Technology, 2-1-6 Etchujima, Koto City, Tokyo 135-8533, Japan \\ ${ }^{* *}$ Center for Energy Studies Gadjah Mada University, Sekip K-1A, Yogyakarta, 50232, Indonesia \\ E-mail: wilson.susanto@mail.ugm.ac.id \\ ***Waseda University, Shinjuku-ku, Tokyo, 169-8555, Japan \\ ****The University of Tokyo, Tokai-mura, Ibaraki, Japan
}

Received: 13 November 2019; Revised: 6 February 2020; Accepted: 30 March 2020

\begin{abstract}
Regarding a severe accident of supercritical water-cooled reactor (SCWR), phase change between subcritical and supercritical conditions is crucial since heat transfer rate changes massively causing a dryout accident. Fundamental knowledge on surface wettability and boiling heat transfer on metals at subcritical conditions under radiation are, thus, important in thermal-hydraulic design and safety analysis of reactor core in light water reactors including a supercritical water-cooled reactor. The radiation induced surface activation (RISA) which enhances wettability and anticorrosive effect on the metal surface was first revealed by authors in 1999. In the earlier studies, significant improvements of surface wettability and boiling heat transfer on oxide film coatedmaterials by the RISA were observed in a room temperature condition. The purpose of this study is to evaluate the effect of oxidized metal and $\gamma$-ray irradiation on metal surface wettability in high pressure and high temperature conditions. In this experiment, the test section was pressurized at $12 \mathrm{MPa}$ with nitrogen gas using pressure vessel and was heated up to temperatures of 20, 150, 200, 250 and 290 centigrade. Two types of material; a stainless-304 and austenitic stainless steel named PNC1520, which is considered as a potential material of fuelcladding tube of the SCWR, were used as specimens. The oxide film on the specimen was formed in supercritical water at 380 centigrade and $22 \mathrm{MPa}$. About $600 \mathrm{kGy}$ Co-60 $\gamma$-ray source was used for irradiation. The results showed that the difference of oxidization on wettability was insignificant at room temperature before $\gamma$-ray irradiation while contact angles on the oxidized specimen decreased at high temperatures. The water growth rate on oxidized material slightly lower compare to non-oxidized material. This result suggests oxide film formation on metal surface plays an important role in surface wettability enhancement by the RISA.
\end{abstract}

Keywords: Supercritical water-cooled reactor, Radiation induced surface activation (RISA), Boiling heat transfer, Wettability, Oxide film

\section{Introduction}

Wettability is the ability of a liquid to maintain contact with a solid surface. Several interesting phenomena related to super-hydrophilic/hydrophobic properties have been reported. It is found that there is an enhancement of both the heat transfer and the critical heat flux using the hydrophobic and hydrophilic mixed surface (Betz et al., 2010). Surface wettability can also be changed by different material types (Phan et al., 2009). From both papers, the heat transfer coefficients become smaller due to governing forces change from a body force to a surface force, nevertheless the interfacial force is predominant in heat transfer. Thus, the contact angle which is an indicator of force balance in wettability was examined. The contact angle equation by Young equation present in eq. (1) and visualize in figure 1.

$\gamma_{S G}-\gamma_{S L}-\gamma_{L G} \cos \theta=0$ 


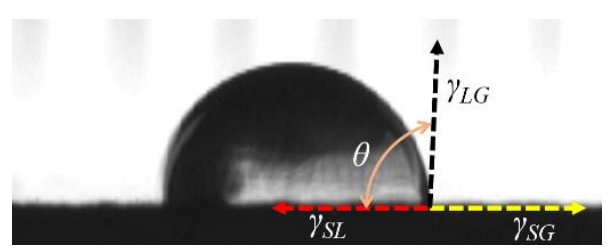

Fig. 1 Droplet contact angle based from Young equation.

As the wettability improvement will boost the heat transfer, a lot of researchers were interested in the enhancement of the wettability of a metal surface by the $\gamma$-ray radiation because of its importance on the heat transfer for fuel pins in light water reactors. Learning about surface wettability and boiling heat transfer on metals at subcritical conditions is a key point to give improvements in thermal-hydraulic design and safety analysis of reactor core. The radiation induced surface activation (RISA) was firstly found by Takamasa et al. (1999). RISA is the phenomenon which activates an oxidized surface to improve the surface wettability remarkably (Takamasa et al., 2004; 2005; 2007). However, the mechanism of the enhancement is still unclear, and the wettability data in subcritical conditions is limited. Otsuka et al. (2015) found that local nano or atomic scale roughness change after the irradiation might enhance the wettability. Other features of the RISA effect were to increase the boiling heat transfer and the anti-corrosive effect.

The RISA mechanism was assumed as shown in Fig. 2, the electrochemical process occurs at the oxide film. It is thought that excited electrons move into the base material and leave holes due to the radiation ray exposure to the metal surface. This activated surface leads to the cathodic and the anodic reaction. $\mathrm{OH}^{-}$from the cathodic reaction reacts with water and make the wettability increases. Therefore, the RISA phenomenon was influenced by the type of materials, oxide film and the cumulative radiation dose. In order to confirm the effect of the oxide metal and $\gamma$-ray irradiation to the wettability enhancement, experiment in high pressure and high temperature conditions was carried out in this study.

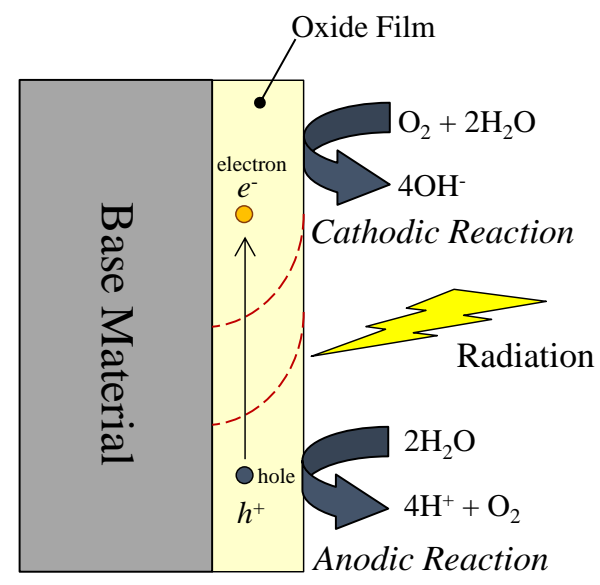

Fig. 2 Assumed RISA mechanism.

\section{Experimental works}

\subsection{Experimental setup and procedures}

A schematic diagram of the apparatus for the wettability experiment at high pressure and high temperature condition shown in Fig. 3. The experimental apparatus consisted of a stainless steel pressure vessel, a single syringe, a water supply pump, a plate light, a High-speed video (HSV) camera, and a LED back-lighting setup. The vessel has a pressureresistance of $15 \mathrm{MPa}$ and the dimension is $50 \mathrm{ID} \times 150 \mathrm{~mm} \mathrm{H} \times 40 \mathrm{~mm} t$. Eight electric heaters were installed inside the pressure vessel wall with a circular pattern to make the heating uniformly. The pressure vessel was heated up to 25,150 , 200, 250 and 290 centigrade. Nitrogen gas was used to pressurize the vessel at $12 \mathrm{MPa}$. The specimen was placed inside the pressure vessel at the center position as shown in Fig. 3. Water is supplied through the syringe (1 mm in diameter) 
installed in the middle hole of the specimen. The water was injected at $100 \mu \mathrm{L} / \mathrm{min}$. The behavior of the water droplet was recorded with the HSV camera through the sapphire glass windows. The LED light was used to obtain the backlighted image.

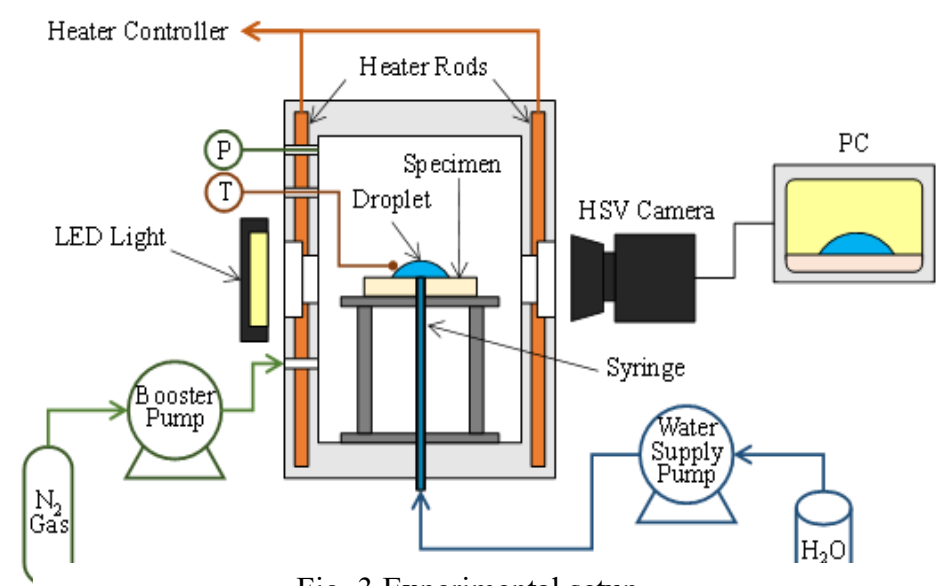

Fig. 3 Experimental setup.

The thermocouples located $10 \mathrm{~mm}$ above the specimen and $20 \mathrm{~mm}$ from the wall of the pressure vessel. In this experiment, two types of material; a stainless-304 (SUS 304) and austenitic stainless steel named PNC1520, which is considered as a potential material of fuel-cladding tube of the supercritical water-cooled reactor (SCWR), were used as specimens. The specimens were polished in advanced respectively using sandpaper $800,1200,2000,4000$ grit, and $\mathrm{Al}_{2} \mathrm{O}_{3}$ powder $(0.3 \mu \mathrm{m})$. Next, cleaned in acetone, ethanol, and pure water using ultrasonic cleaner consecutive to sterilize from contamination. The typical chemical weight percent compositions of the materials shown in Table 1.

Table 1 Typical chemical weight percent compositions of the material.

\begin{tabular}{|c|c|c|c|c|c|l|l|l|l|l|l|}
\hline Material & $\mathrm{C}$ & $\mathrm{Si}$ & $\mathrm{Mn}$ & $\mathrm{P}$ & $\mathrm{S}$ & $\mathrm{Ni}$ & $\mathrm{Cr}$ & $\mathrm{Co}$ & $\mathrm{Ti}$ & $\mathrm{Mo}$ & $\mathrm{Nb}$ \\
\hline SUS304 & 0.06 & 0.56 & 0.91 & 0.036 & 0.003 & 8.05 & 18.17 & 0.20 & - & - & - \\
\hline PNC1520 & 0.058 & 0.81 & 1.75 & 0.030 & 0.002 & 20.06 & 14.83 & $<0.01$ & 0.24 & 2.48 & 0.10 \\
\hline
\end{tabular}

The dimension of all specimens was $16 \times 16 \times 3 \mathrm{~mm}$ with a $1 \mathrm{~mm}$ hole in the middle of specimens. Figure 4 shows the oxide film on specimens. Specimens were immersed in supercritical water at 380 centigrade and 22 MPa for 2 weeks (336 hours). Because surface roughness has a strong correlation to the surface wettability, surface roughness was evaluated for all oxidized materials. Using confocal microscopy measurement, 1 line was taken as the sample of the whole material. The surface of the oxidized material is shown in Fig. 5, the average surface roughness of the sample line is $2.25 \mu \mathrm{m}$ with $0.88 \mu \mathrm{m}$ deviation. The average value of all oxidized materials surface roughness is $5.13 \mu \mathrm{m}$ with $0.83 \mu \mathrm{m}$ deviation. Our group tried to reduce the surface roughness of oxidized materials through polishing with sandpaper 4000 grit, however, the oxidized film peeled as soon the surface polished. Based on this experience, the surface roughness in this experiment was ignored. Irradiation was performed with a Co-60 $\gamma$-ray source at Institute for Integrated Radiation and Nuclear Science, Kyoto University and the cumulative radiation exposure was approximately $600 \mathrm{kGy}$. 


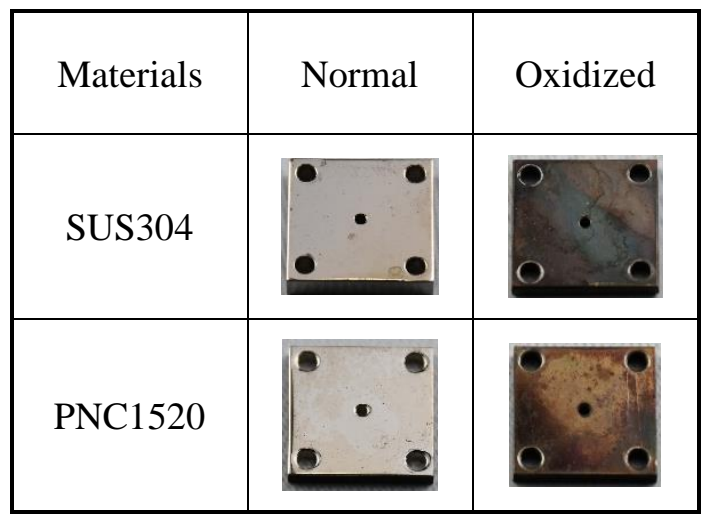

Fig. 4 Pictures of the specimen surface.

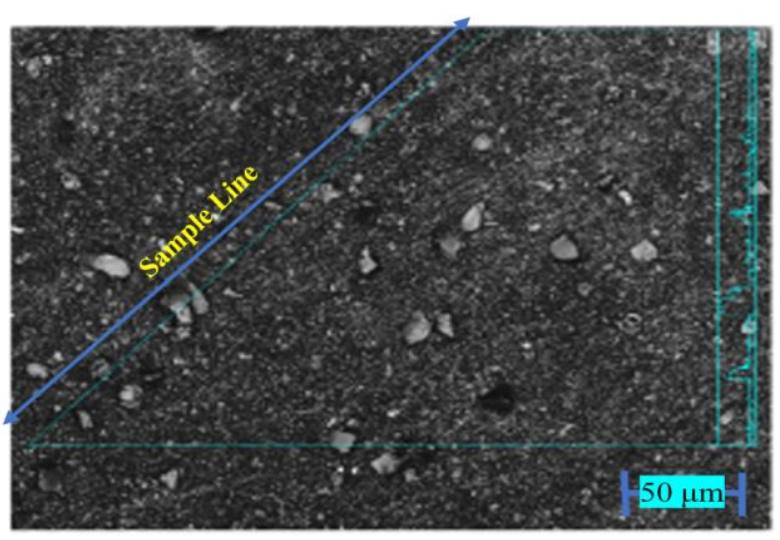

a. Surface roughness of oxidized material
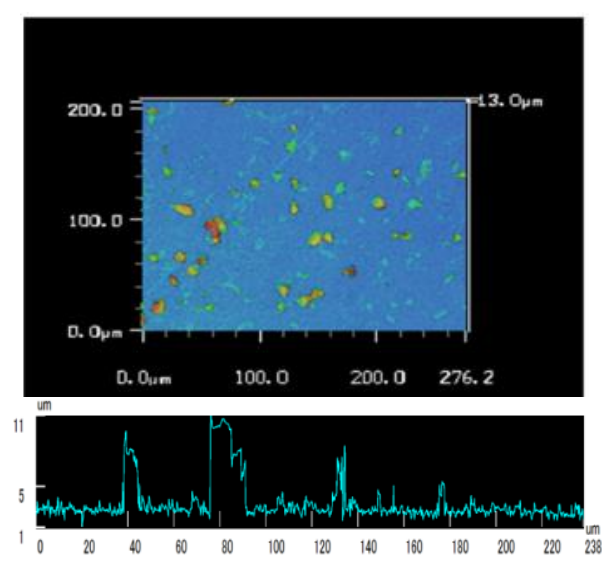

b. Detailed surface roughness number

Fig. 5 Surface roughness oxidized material using confocal microscopy.

In the present experiment, when water infused from the bottom, the droplet forms in a spherical shape. Using this experimental setup only the advancing contact angle can be measured. However, if the capillary number of a droplet is low, the advancing contact angle is assumed as static contact angle, therefore capillary number was checked as the fluctuation parameter. The average capillary number of this experiment is $8.9 \times 10^{-6}$ which indicated a low capillary number (less than $10^{-5}$ ). Figure 6 illustrates the continuous droplet growth in the upper direction and in the side direction (advancing contact angle). Once it meets the equilibrium point, the water evenly expands to all directions.

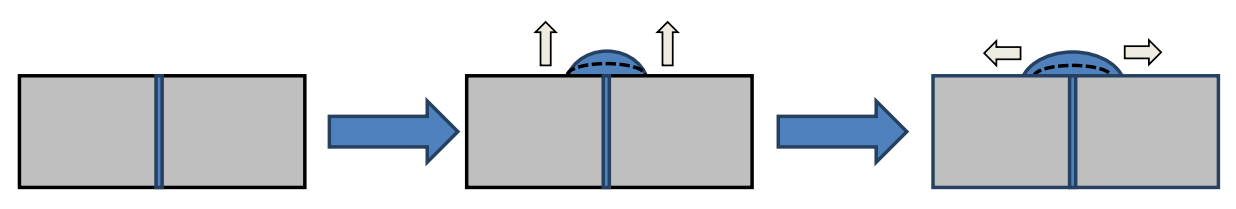

Fig. 6 Droplet deformation.

During the water injection at a designated pressure and temperature, the video of water was recorded with the HSV camera. The contact angle, which is an indicator of macroscopic wettability, was measured by image-processing. The water droplet stabilizes after 4 seconds or more with the maximum deviation of 2 degrees, thus the contact angle was taken at 5 seconds after water came out from the injection hole. 


$$
\begin{aligned}
& \tan \theta=\frac{2 h}{w} \\
& 2 \theta=2 \tan ^{-1} \frac{2 h}{w}
\end{aligned}
$$

Where:

$$
\begin{aligned}
\theta & =\text { Contact angle } \\
h & =\text { Droplet height } \\
w & =\text { Droplet diameter }
\end{aligned}
$$

The height and diameter of the droplet were measured to calculate the contact angle using the equation (3) above. The height and diameter of the droplet were shown in Fig. 7;

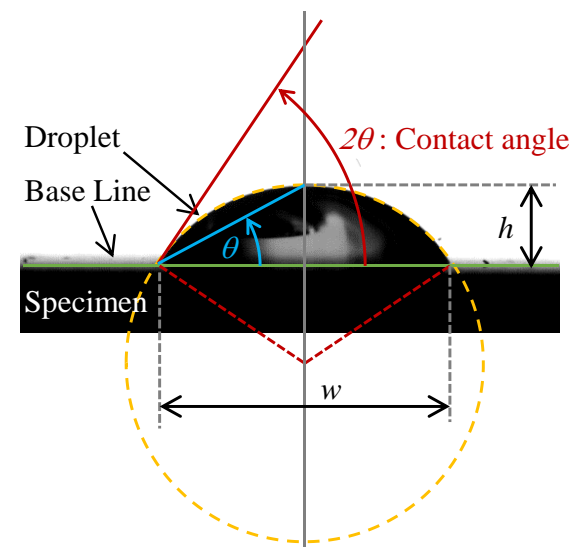

Fig. 7 Contact angle derivation.

The water growth rate in 5 seconds also evaluated to measure the evaporation rate. Our group assumed the contact area of liquid and metal is a circular shape, therefore the water volume equation will be:

$$
V=\frac{2}{3} \pi\left(\frac{w}{2}\right)^{2} h
$$

\subsection{Experimental Results}

To delineate the effect of RISA on surface wettability on SUS 304, PNC 1520, oxidized SUS 304 and oxidized PNC 1520 at high temperature and high-pressure conditions. The experiment was conducted under $12 \mathrm{MPa}$ and temperature 25 to 290 centigrade. Using equation (4), the volume of the droplet was measured every 1 second and volume growth rate (Q) was obtained through the average value of growth rate in 5 seconds. Figure 8 and fig. 9 show the correlation of water growth rate and temperature on non-oxidized and oxidized material. Even though the pump injection was set at $100 \mu \mathrm{L} / \mathrm{min}$ or $1.67 \mu \mathrm{L} / \mathrm{s}$, the actual pump injection is about $0.8 \mu \mathrm{L} / \mathrm{s}$. The water growth rate was increased at 150 and $200{ }^{\circ} \mathrm{C}$ and gradually decrease as the temperature increase due to evaporation. Both figures show that the water growth rate on oxidized material are slightly lower compare to non-oxidized material, though the reduction needs more accuracy analysis in the future. However, this result also corroborates the experiment had a low capillary number due to the low volume growth rate. 


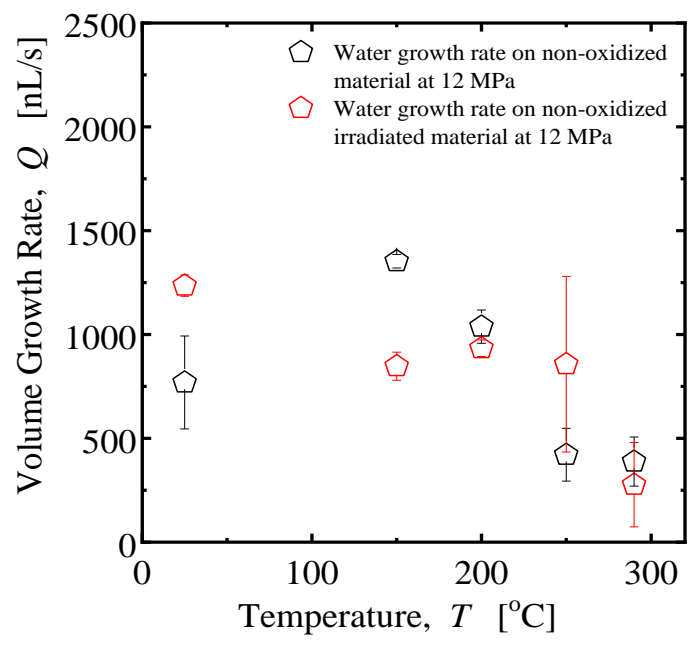

Fig. 8 Correlation water growth rate and temperature on non-oxidized material.

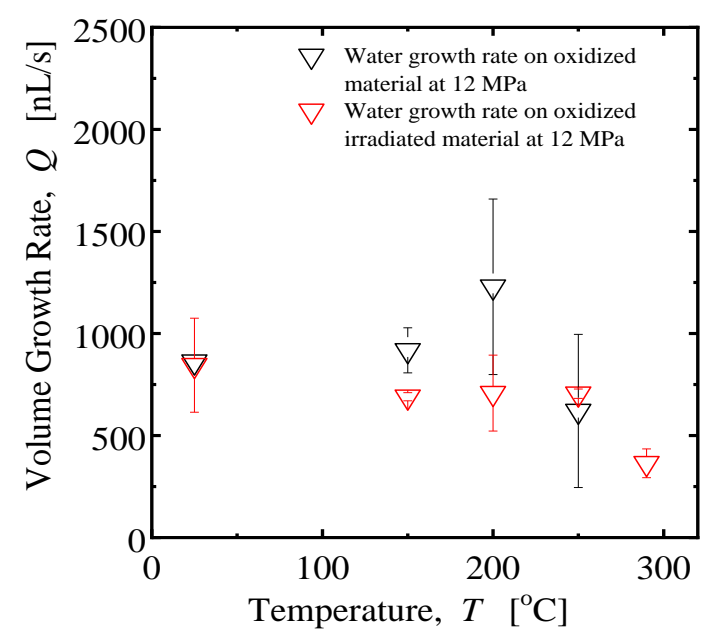

Fig. 9 Correlation water growth rate and temperature on oxidized material.

\section{a. Effect of $\boldsymbol{\gamma}$-ray radiation to the surface wettability for normal SUS 304 and PNC 1520 materials.}

The contact angle was measured every 0.5 seconds for 5 seconds to check the deviation. Figure 10 indicates there is a bigger fluctuation in the initial condition compare to the final condition, the deviation in the initial condition is around $2^{\circ}$, in addition, the final condition is around $1^{\circ}$ because the droplet was not fully formed yet. On this basis, the contact angle was taken at 5 seconds when the least deviation was observed. 


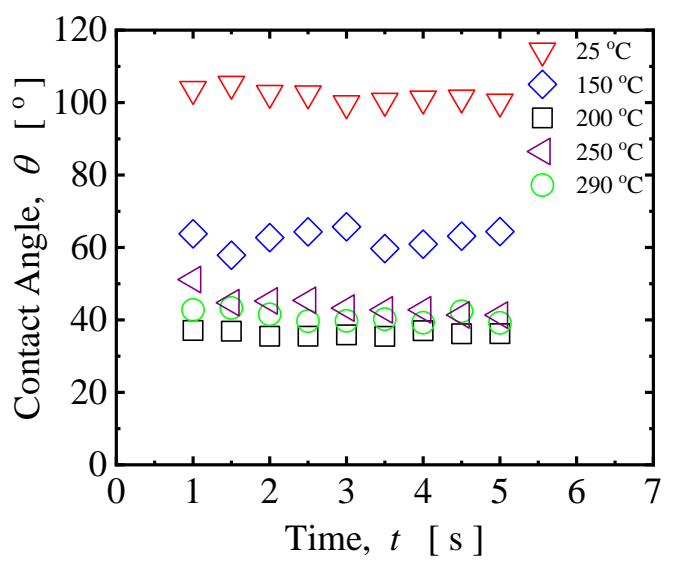

Fig. 10 Contact angle time series on SUS materials.

Figures 11 and 12 display the effect of temperature on the contact angle for normal SUS 304 and PNC 1520 materials before and after $\gamma$-ray radiation. Both figures illustrate the same tendencies; for the normal non-irradiated specimen, the contact angle decreases as the temperature increases while the irradiated specimen showed the significant development of wettability at room temperature condition. Wettability improvement of irradiated specimens slowly decreases as the temperature increases. This phenomenon can be explained that the surface tension of water was decreased as molecules becomes more active at the higher temperature thus the contact angle become small. Under hydrophilic condition it seems that there is some limitation to the improvement to the RISA, therefore the effect was insignificant.

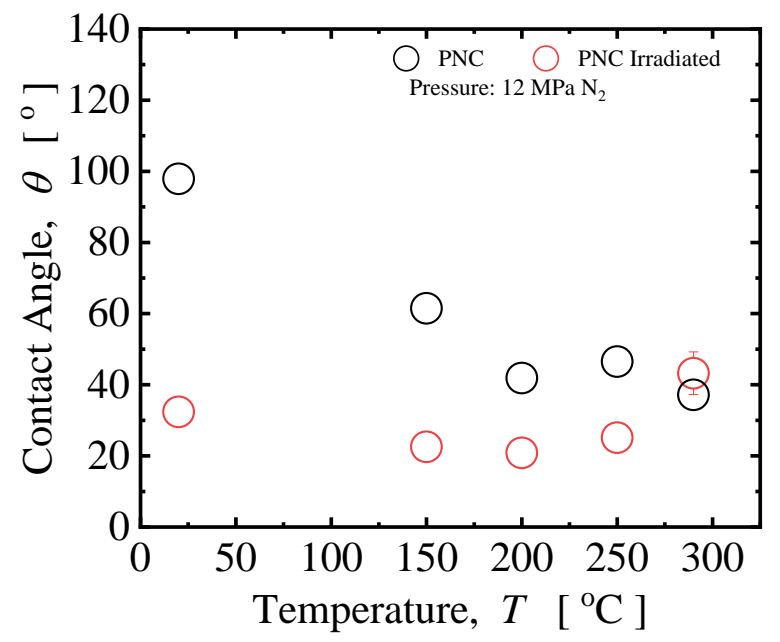

Fig. 11 Contact angle on SUS 304 before and after $\gamma$-ray radiation. 


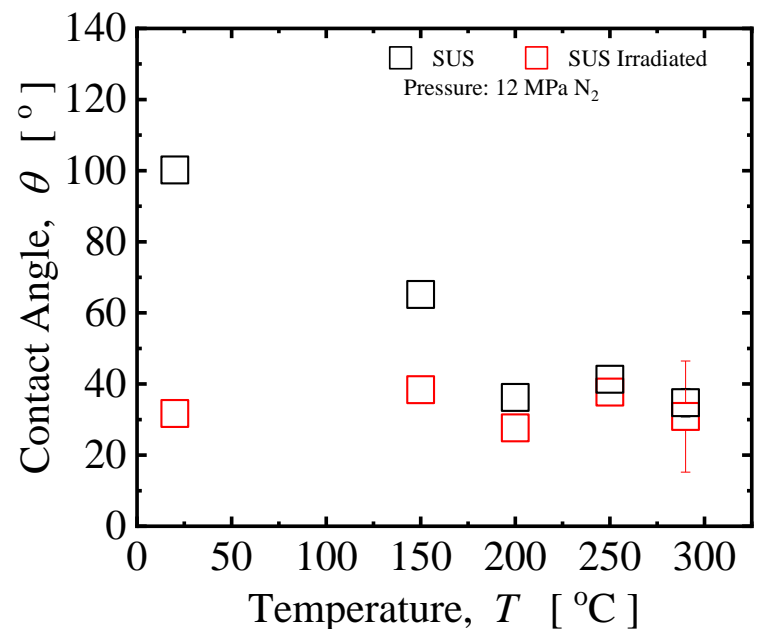

Fig. 12 Contact angle on PNC 1520 before and after $\gamma$-ray radiation.

\section{b. Effect of oxide film to the surface wettability on the SUS 304 and PNC 1520 materials.}

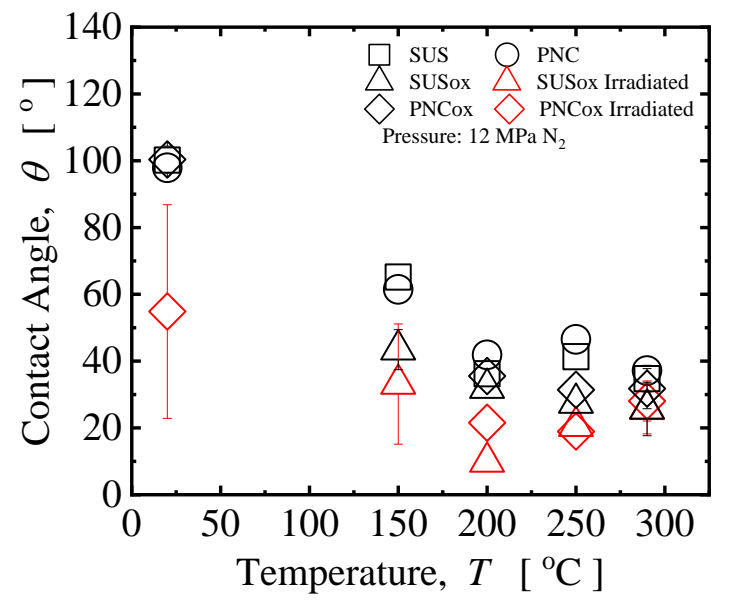

Fig. 13 Effect of oxide film to the contact angle on the SUS 304 and PNC 1520 metals surface.

Figure 13 indicated the effect of the oxide film on the contact angle for the SUS 304 and PNC 1520 materials with and without oxide film. For normal specimens, oxide film increases the wettability, the most significant improvements are shown at 150 and 250 centigrade. After irradiated the contact angle oxidized specimen considerably decreases at room temperature condition, while the improvement of contact angle slightly decreases at the higher temperature. It is assumed that the $\mathrm{OH}^{-}$formation generated easily on material with oxide film compare to the material without oxide film. It suggests that a detailed investigation to clarify the macroscopic structure of oxidized materials is needed to examine the $\mathrm{OH}^{-}$formation before and after $\gamma$-ray irradiation.

\section{Conclusions}

In this paper, the RISA effect was observed through contact angle which is an indicator of macroscopic surface wettability on the SUS 304 and PNC 1520 metals surface at 25 to 290 centigrade. The results are summarized as follows:

(1) The water growth rate on oxidized material slightly lower compare to non-oxidized material, though the reduction needs more accuracy analysis in the future.

(2) The enhancement on the contact angle due to RISA effect was achieved for all range temperature regardless any 
type of specimen, while the significant effect shows at room temperature condition and the effect decreases as the temperature increases. Significant difference due to the RISA effect was not confirmed over $250{ }^{\circ} \mathrm{C}$.

(3) The oxide film which formed under supercritical condition does not contribute wettability enhancement significantly, although the oxide film formation on metal surface plays an important role in surface wettability enhancement by the RISA.

An experiment to clarify the macroscopic structure of oxidized materials will be needed to examine the $\mathrm{OH}^{-}$ formation before and after $\gamma$-ray irradiation in the future.

\section{Acknowledgement}

The authors are very thankful to Mr. T. Miyoshi of Waseda University, Mrs. Y. Ohkubo and Professor T. Takamasa of Tokyo University of Marine Science and Technology for their collaboration in conducting the experiment. The gammaray irradiation was carried out using the QST (Takasaki) Co-60 facility supported by the Inter-University Program for the Joint-use of JAEA/QST Facilities, Nuclear Professional School, School of Engineering, the University of Tokyo (proposal no. 18017 and 19018). Part of this work was supported by MEXT Innovative Nuclear Research and Development Program Grant Number JPMXDO215657614.

\section{References}

Betz, A. R., Xu, J., Qiu, H., and Attinger, D., Do surface with mixed hydrophilic and hydrophobic area enhance pool boiling? Appl. Phys. Lett., Vol. 97 (2010), pp. 141909-1- 141909-3.

Ding, M., and Kantzas, A., Capillary Number Correlations for Gas-Liquid Systems. Petroleum Society of Canada (2004), DOI:10.2118/2004-062.

Hazuku, T., Hirose Y., Hayashi T., Pollman A., Fukuhara Y., and Takamasa T., Effect of Radiation Irradiation on Surface Wettability under High-temperature and High-pressure Environment, Transactions of the Japan Society of Mechanical Engineers (B) (2009) 75-758, 1911-1916.

Kikuchi, T., Hirose Y., Hazuku T., and Takamasa T., Effect of Radiation Induced Surface Activation on Surface Wettability in High-temperature Condition (2007), ICONE15-10316.

Otsuka, T., Isotani, T., Nakayama K., Morishige N., Hashizume K., Sugisaki M., and Tanabe T., On the Wettability Change of Oxidized and Gamma Irradiated Zircaloy, water reactor fuel performance meeting (2015), pp.1236.

Phan, H. T., Caney, N., Marty, P., Colasson, S., and Gavillet, J., How does surface wettability influence nucleate boiling? C.R. Mecanique, Vol. 337 (2009), pp. 251-259. Phan, H. T, Cabeny, N., Marty, P., Colasson, S., \& Gavillet, J.

Takamasa T., Hazuku T., Mishima K., Okamoto K., and Imai Y., Surface Wettability Caused by Radiation Induced Surface Activation, Thermal Science \& Engineering, Vol. 12, No. 2, pp. 39-44, 2004.

Takamasa, T., Hazuku T., Okamata K., Mishima K., and Furuya M., Radiation induced surface activation on Leidenfrost and quenching phenomena, Experimental Thermal and Fluid Science, Volume 29 (2005), p.267-274 